\title{
Special issue on biopacemaking: clinically attractive, scientifically a challenge
}

\author{
Jacques M. T. de Bakker · Antonio Zaza
}

Received: 11 January 2007 / Accepted: 12 January 2007 / Published online: 6 February 2007

(C) International Federation for Medical and Biological Engineering 2007

This special issue gives an overview of the current state-of-the-art of creating a bio-engineered pacemaker. The subject has potential clinical interest. Indeed, electronic pacemakers currently available have several limitations, among which inadequate rate adaptation to physiological needs, problems related to the stimulating and sensing leads and infection of the pacemaker pocket, which might be overcome by a biopacemaker. Generation of a bio-pacemaker has also scientific interest, because it may answer the longstanding question of whether the complex structure of the sinus node is indeed a prerequisite for reliable pacemaking, or simpler structures might work as well.

Knowledge of normal pacemaker physiology provides the ground for the development of bio-pacemakers. Various ionic currents contribute to sinoatrial (SA) node pacemaking; moreover, the sinus node comprises morphologically and functionally distinct cell types, with different intrinsic rates and response to

\section{J. M. T. de Bakker ( $\square)$}

Department of Experimental Cardiology,

Heart Failure Research Center, Academic Medical Center, Meibergdreef 9, 1105AZ Amsterdam, The Netherlands

e-mail: j.m.debakker@amc.uva.nl

\section{J. M. T. de Bakker}

The Heart Lung Center, University Medical Center, Utrecht, The Netherlands

\section{J. M. T. de Bakker}

The Interuniversity Cardiology Institute of the Netherlands, Utrecht, The Netherlands

\section{A. Zaza}

Dipartimento di Biotecnologie e Bioscienze,

Università di Milano-Bicocca, Milano, Italy

e-mail: antonio.zaza@unimib.it autonomic agonists. As outlined by Opthof [11], these differences are relevant to the width and stability of autonomic modulation of sinus rate. The question may be asked of whether such complexity, probably the result of evolutionary adaptations, would also be required to create a bio-pacemaker. For reasons of practicality, the strategies proposed thus far have adopted a conservative "one channel" approach; however, as genetic manipulation techniques improve, reports of bio-pacemakers based on combinations of mechanisms start appearing in the literature [3].

Current approaches to bio-pacemaker generation have developed along two main lines. The first aims to induce pacemaker activity in normally quiescent ("working") myocardium. The second involves myocardial implant of exogenous cells, engineered to sustain pacemaker activity ("cell-based" approach), once electrically connected to the host myocardium.

Pacemaking can be induced in working myocardial cells by modification of their pattern of expression of membrane currents. The required genetic modification is usually carried out by gene transfer to the site of interest. This can be achieved theoretically by direct transfection of a plasmid incorporating the gene, or by infecting the tissue with a viral vector containing it. In practical terms, only the viral infection provides adequate transduction efficiency and is universally adopted. Nonetheless, the infection procedure involves a number of technical and safety problems. The replication-deficient adenovirus is a safe and practical vector; however, because the gene is not incorporated into the genome, its expression is only transient. Retroviruses, like the widely used lentivirus, incorporate the added gene into the genome, which results in stable gene expression. However, genomic transduction 
carries potential carcinogenic risk, which might make this type of vector less suitable for therapeutic use. Such problems have prompted the development of cell-based approaches, in which pacemaker function is intrinsic to the implanted cell, or can be obtained by genetic modification prior to implant in the host myocardium.

In the cell-based approach, several strategies have been proposed. In one case spontaneously beating clusters of myocytes derived from human embryonic stem cells (hESCs) were directly used as pacemaker elements [15]. However, once implanted, these cells could further differentiate into quiescent elements, thus compromising pacemaker stability. Another, more promising, approach is based on in vitro genetic modification of exogenous cells, originally devoid of pacemaker activity, which are stably transduced with a gene encoding the current of interest. Once implanted, the modified cells electrically couple to the surrounding myocardium, and modulate its electrical activity $[2,12]$. Cell-to-cell coupling is mediated by connexins, protein channels that allow ionic current flow between adjacent cells. Connexins are at hand in many cell-types, including stem cells, which can successfully couple to cardiac myocytes. Success of the cell-based approach depends on the possibility of avoiding immunological rejection of the implant; thus an autologous origin of the implanted cells is highly desirable. Stem cells may be particularly suitable for generating a bio-pacemaker because they can be autologous and they replicate, thus allowing amplification of the cell population. An alternative may be the development of replicating celllines, engineered to achieve immunocompatibility.

To create a bio-pacemaker, the following strategies are currently followed: (1) suppression of repolarizing currents to unmask latent pacemaker currents in normally quiescent myocardial cells; (2) over-expression of a pacemaker (depolarizing) current in electrically quiescent cells to convert them into pace-making elements; (3) modulation of the expression of receptors involved in the regulation of pacemaker currents [5].

The first approach, a pioneering one in the field of bio-pacemakers, relies on the idea that ventricular "working" myocardium has latent pacemaker activity, but spontaneous depolarization is normally suppressed by a large repolarizing conductance, available at diastolic potential. Such a conductance is provided by the "inward rectifier" potassium current $I_{\mathrm{K} 1}$, known for its strong expression in electrically quiescent cells of the atrial and ventricular working myocardium, but virtually absent from the AV and SA node. Therefore, suppression of $I_{\mathrm{K} 1}$ is a putative approach for creating a bio-pacemaker. The group of Marban [9] provided a proof of this concept by using a dominant-negative Kir2.1 construct, packaged into an adenoviral vector. Once infected with the vector in vivo, ventricular myocardium showed $80 \% I_{\mathrm{K} 1}$ suppression and developed automatic activity [9]. Although conceptually innovative, such an approach is encumbered by the problems related to all viral transduction methods; moreover, strict delimitation of the infection site is difficult and diffusion of $I_{\mathrm{K} 1}$ suppression throughout the ventricle may entail pro-arrhythmic risk.

With regard to over-expression of depolarizing currents, much attention has been given to the main depolarizing current that induces spontaneous activity in the SA node, the funny current $I_{\mathrm{f}}$, which is mediated by a family of hyperpolarization-activated cyclic nucleotide-gated (HCN) channels. This area has been pioneered by Rosen et al. [13], whose review in this issue summarizes the evolution of the concept and the results obtained. These authors used $\mathrm{HCN} 2$ as the $I_{\mathrm{f}}$ encoding isoform because the resulting current kinetics are more favorable than with HCN4 and its cAMP responsiveness is greater than that of $\mathrm{HCN} 1$. These investigators initially showed suitability of $I_{\mathrm{f}}$ overexpression by injecting $\mathrm{HCN} 2$ encoding adenoviral vectors into the left atrium or the left bundle branch of intact dog hearts. Both injection sites proved to be successful in generating an ectopic rhythm. In addition, the experiments also proved that pacemaker activity generated by expression of $\mathrm{HCN} 2$ was autonomically regulated. To overcome the problems related to the adenoviral infection method, the same group developed a cell-based approach. Human mesenchymal stem cells (hMSC), loaded with the HCN2 gene, were injected epicardially into the left ventricular free wall and resulted into an idioventricular rhythm at the injection site. This rhythm was significantly faster than the escape rhythm following AV nodal ablation, thus providing efficient pacemaker activity. Recent studies also explored the feasibility to convert quiescent ventricular myocytes into pacemakers using somatic cell fusion [4, 8]. Chemically induced fusion between myocytes and syngeneic fibroblasts that had been engineered to express pacemaker ion channels, has been attempted. The advantage of this approach, with respect to classical cell-based therapy, is that the gapjunctional coupling between donor cells and host myocardium, which might be sub-optimal or unstable in time, is avoided.

Interestingly, a cell-based approach has also been proposed as a mean to down-regulate heart rate. De Boer et al. [2] reduced beating rate of spontaneously active neonatal rat cardiomyocytes by co-culturing them with $I_{\mathrm{K} 1}$ overexpressing human embryonic 
kidney cells (HEK, transduced with Kir2.1 gene). These investigators also showed that the influence of Kir2.1 expressing cells on beating rate could be lessened by the application of $\mathrm{BaCl}_{2}$, that blocks $I_{\mathrm{K} 1}$. Since pacemaker down-regulation occurred through electronic interaction between the two cell types, this result also implies that efficient connexin-mediated cell-tocell coupling spontaneously develops between HEK cells and ventricular myocytes.

Recent evolutions in bio-pacemaking techniques involve the expression of "synthetic" pacemaker channels, obtained by modification of genes originally encoding non-pacemaker currents. The rationale of this approach is the concern that co-assembly of added HCN proteins with those naturally expressed by the cell may result in unpredictable channel properties. To generate a synthetic pacemaker channel, Kashiwakura et al. [7] converted the depolarization-activated potassium channel Kv1.4 into a hyperpolarizationactivated non-selective channel by 4-point-mutations. The properties of the synthetic channel were similar to those of $\mathrm{HCN}$ ones, but co-assembly between endogenous and added proteins was avoided.

A requirement for successful propagation of pacemaker activity is an appropriate match between the pacemaker generator properties and the electrical load imposed by the tissue to be excited. Electrical-coupling is required for propagation between pacemaker and follower cells but, if the load is excessive, it may arrest the pacemaker by clamping pacemaker cells to hyperpolarized resting membrane potentials. The SA node has special means to circumvent this problem, including expression of an hyperpolarization-activated depolarizing current $\left(I_{\mathrm{f}}\right)$ [10] and a complex architecture of the node-atrium interface $[1,6]$. In the case of bio-pacemakers, the interface architecture can be hardly controlled; thus, for their development, prediction of the interplay between polarizing and depolarizing currents and quantitative estimates of the required generator size may be necessary. As reviewed by Wilders in this issue [14], accurate computer models of the SA node activity, now available, may help in understanding how depolarizing and repolarizing currents interact and respond to perturbing conditions. The problem of the match between generator and load is illustrated in this issue by Joyner et al. [6]. These investigators addressed this problem with a mixed approach in which SA electrical activity, generated by a numerical model, was electrically coupled through a variable resistor to a real atrial myocyte. This allowed to test how coupling resistance may affect the pacemaker-load interaction and to obtain a quantitative evaluation of the conditions required for propagated pacemaking [6].

As highlighted in this issue, research in the field of bio-pacemaking is blooming. Nonetheless, in light of the performance and safety of the electronic pacemakers now available, development of a better alternative is an extremely demanding task. It yet has to be proven that the bio-pacemaker surpasses its electronic counterpart with regard to adaptability to physiological requirements of the body and longevity. While potentially effective pacemaking strategies have been identified, the development of genetic engineering methods suitable to implement them with safety and stability remain a considerable challenge. The possibility of uncontrolled gene expression, carcinogenic risk of viral vectors affording stable transduction and immune rejection of implants are among the problems that need to be solved before bio-pacemaking can be considered for clinical use. Moreover, ventricular re-synchronization, a major advancement of artificial pacemaking, may be difficult to achieve with biopacemakers.

Despite these concerns, bio-pacemaking seems more easily achievable than other potential applications of cardiac cell therapy. This is because bio-pacemaking aims to restore a single function with a well-defined mechanism; it requires myocardial homing of a limited number of cells and a localized intervention. Development of bio-pacemakers may be an ideal challenge for the approach typical of bioengineering, based on a close interaction between expertise in biophysics, molecular and cell biology.

\section{References}

1. Anghel TM, Pogwizd SM (2006) Creating a cardiac pacemaker by gene therapy. Med Biol Eng Comput; MBEC 514

2. de Boer TP, van Veen TA, Houtman MJ, Jansen JA, van Amersfoorth SC, Doevendans PA, Vos MA, van der Heyden MA (2006) Inhibition of cardiomyocyte automaticity by electrotonic application of inward rectifier current from Kir2.1 expressing cells. Med Biol Eng Comput 44:537-542

3. Cho HC, Kashiwakura Y, Marban E (2005) Conversion of non-excitable cells to self-contained biological pacemakers. Circulation 112(17):II-307

4. Cho HC, Kashiwakura Y, Marban E (2005) Creation of a biological pacemaker by cell fusion. Circulation 112(17): II-307

5. Edelberg JM, Aird WC, Rosenberg RD (1998) Enhancement of murine cardiac chronotropy by the molecular transfer of the human beta2 adrenergic receptor cDNA. J Clin Invest 101:337-343

6. Joyner RW, Wilders R, Wagner MB (2006) Propagation of pacemaker activity. Med Biol Eng Comput; MBEC 389 
7. Kashiwakura Y, Cho HC, Barth AS, Azene E, Marban E (2006) Gene transfer of a synthetic pacemaker channel into the heart: a novel strategy for biological pacing. Circulation 114:1682-1686

8. Marban E, Cho HC (2006) Creation of a biological pacemaker by gene- or cell-based approaches. Med Biol Eng Comput; MBEC 559

9. Miake J, Marban E, Nuss HB (2003) Functional role of inward rectifier current in heart probed by Kir2.1 overexpression and dominant-negative suppression. J Clin Invest 111:1529-1536

10. Noble D, Denyer JC, Brown HF, DiFrancesco D (1992) Reciprocal role of the inward currents $\mathrm{Ib}, \mathrm{Na}$ and If in controlling and stabilizing pacemaker frequency of rabbit sino-atrial node cells. Proc R Soc Lond B 250:199-207

11. Opthof T (2006) Embryological development of pacemaker hierarchy and membrane currents related to the function of the adult sinus node. Implications for autonomic modulation of biopacemakers. Med Biol Eng Comput; MBEC 413
12. Potapova I, Plotnikov A, Lu Z, Danilo P Jr, Valiunas V, Qu J, Doronin S, Zuckerman J, Shlapakova IN, Gao J, Pan Z, Herron AJ, Robinson RB, Brink PR, Rosen MR, Cohen IS (2004) Human mesenchymal stem cells as a gene delivery system to create cardiac pacemakers. Circ Res 94:952-959

13. Rosen MR, Brink PR, Cohen IS, Robinson RB (2006) Biological pacemakers based on If. Med Biol Eng Comput; MBEC 335

14. Wilders R (2006) Computer modelling of the sinoatrial node. Med Biol Eng Comput; MBEC 509

15. Xue T, Cho HC, Akar FG, Tsang SY, Jones SP, Marban E, Tomaselli GF, Li RA (2005) Functional integration of electrically active cardiac derivatives from genetically engineered human embryonic stem cells with quiescent recipient ventricular cardiomyocytes: insights into the development of cell-based pacemakers. Circulation 111:11-20 